\title{
Solution of a class of the first kind singular integral equation with multiplicative Cauchy kernel
}

\begin{abstract}
In the present paper, we give the exact solutions of a singular equation with logarithmic singularities in two classes of functions and construct formulae for the approximate solutions.
\end{abstract}

1. Introduction. Let us consider a singular integral equation of the form

(1) $\frac{1}{\pi^{2}} \iint_{D} \frac{\varphi(\xi, \eta)}{(\xi-x)(\eta-y)} d \xi d \eta=f(x, y) \ln \frac{1-x}{1+x} \ln \frac{1-y}{1+y},(x, y) \in D$,

where $D=(-1,1) \times(-1,1), f(x, y)$ is a given Hölder continuous function in $\bar{D}$, and $\varphi(x, y)$ is an unknown function. The equation (1) has applications in the theory of aeroelasticity [1].

Note that the equation without logarithmic singularities was many times considered in different classes of functions. In the literature the solutions of the equation (1) in bounded domains $[2,5,6,9]$ as well as unbounded $[3,4,7,8,10]$, are known for both single and multiple integrals.

Let us introduce the function classes that will be used here.

2000 Mathematics Subject Classification. 45E05, 65R20.

Key words and phrases. Singular integral equations, Cauchy-type kernel, multiplicative kernel, logarithmic singularities. 
Definition 1. We write $\varphi(x, y) \in h(-1,1) \times h(-1,1)$, if it satisfies the Hölder inequality

$$
\left|\varphi(x, y)-\varphi\left(x^{\prime}, y^{\prime}\right)\right| \leq K_{1}\left|x-x^{\prime}\right|^{\mu_{1}}+K_{2}\left|y-y^{\prime}\right|^{\mu_{2}},
$$

where $0<\mu_{1}, \mu_{2} \leq 1$, and $K_{1}, K_{2}>0$ are constants independent of the choice of points $(x, y),\left(x^{\prime}, y^{\prime}\right) \in \bar{D}$.

Definition 2. We write $\varphi(x) \in h_{0}$, if it satisfies the Hölder inequality in each point of the interval $(-1,1)$, and has the following representation

$$
\varphi(x)=\frac{\varphi_{1}^{*}(x)}{(1+x)^{\alpha_{1}}}, \quad \varphi(x)=\frac{\varphi_{2}^{*}(x)}{(1-x)^{\alpha_{2}}}
$$

in a neighborhood of the points $x=-1, x=1$ respectively, with $0 \leqslant$ $\alpha_{1}, \alpha_{2}<1$, and $\varphi_{1}^{*}(x), \varphi_{2}^{*}(x)$ satisfying the Hölder inequality in the interval $[-1,1]$.

Definition 3. We write $\varphi(x, y) \in h_{0} \times h_{0}$, if it satisfies the Hölder inequality (2) in each interior point of the set $D$, and has the following representation

$$
\begin{array}{ll}
\varphi(x, y)=\frac{\varphi_{1}^{*}(x, y)}{(1+x)^{\alpha_{1}}}, & \varphi(x, y)=\frac{\varphi_{2}^{*}(x, y)}{(1-x)^{\alpha_{2}}}, \\
\varphi(x, y)=\frac{\varphi_{3}^{*}(x, y)}{(1+y)^{\alpha_{3}}}, & \varphi(x, y)=\frac{\varphi_{4}^{*}(x, y)}{(1-y)^{\alpha_{4}}}
\end{array}
$$

in a neighborhood of points of the lines $x=-1, x=1, y=-1, y=1$, respectively, with $0 \leqslant \alpha_{k}<1$, and $\varphi_{k}^{*}(x, y)$ belonging to the class $h(-1,1) \times$ $h(-1,1)$, for $k=1, \ldots, 4$.

\section{Exact solution in the class $h_{0} \times h_{0}$.}

Theorem 1. Let the function $f(x, y) \in h(-1,1) \times h(-1,1)$. Then the general solution of the equation (1) in the class $h_{0} \times h_{0}$ has the form

$$
\varphi(x, y)=\frac{1}{\sqrt{\left(1-x^{2}\right)\left(1-y^{2}\right)}} R(f ; x, y)+\frac{\gamma_{1}(x)}{\sqrt{1-y^{2}}}+\frac{\gamma_{2}(y)}{\sqrt{1-x^{2}}}
$$

where

$$
R(f ; x, y)=\frac{1}{\pi^{2}} \iint_{D} \frac{\sqrt{\left(1-\xi^{2}\right)\left(1-\eta^{2}\right)} f(\xi, \eta) \ln \frac{1-\xi}{1+\xi} \ln \frac{1-\eta}{1+\eta}}{(\xi-x)(\eta-y)} d \xi d \eta
$$

and $\gamma_{1}(x), \gamma_{2}(y)$ are arbitrary functions from the class $h_{0}$. 
If the solution $\varphi(x, y)$ satisfies the conditions

$$
\begin{aligned}
& \frac{1}{\pi} \int_{-1}^{1} \varphi(x, \eta) d \eta=g(x),-1<x<1, \\
& \frac{1}{\pi} \int_{-1}^{1} \varphi(\xi, y) d \xi=h(y),-1<y<1,
\end{aligned}
$$

where $g(x)$ and $h(x)$ are given functions of the class $h_{0}$ such that

$$
\frac{1}{\pi} \int_{-1}^{1} g(\xi) d \xi=\frac{1}{\pi} \int_{-1}^{1} h(\eta) d \eta=A,
$$

then the equation (1) has the unique solution given by the following formula:

$$
\begin{aligned}
& \varphi(x, y)=\frac{R(f ; x, y)}{\sqrt{\left(1-x^{2}\right)\left(1-y^{2}\right)}}+\frac{g(x)}{\sqrt{1-y^{2}}}+\frac{h(y)}{\sqrt{1-x^{2}}} \\
& -\frac{A}{\sqrt{\left(1-x^{2}\right)\left(1-y^{2}\right)}} \text {. }
\end{aligned}
$$

Proof. Denoting

$$
\begin{aligned}
& \Psi_{1}(x, y)=\frac{1}{\pi} \int_{-1}^{1} \frac{\varphi(x, \eta)}{\eta-y} d \eta, \\
& \Psi_{2}(x, y)=\frac{1}{\pi} \int_{-1}^{1} \frac{\varphi(\xi, y)}{\xi-x} d \xi,
\end{aligned}
$$

one can express the equation (1) in the form

$$
\frac{1}{\pi} \int_{-1}^{1} \frac{\Psi_{1}(\xi, y)}{(\xi-x)} d \xi=f(x, y) \ln \frac{1-x}{1+x} \ln \frac{1-y}{1+y},
$$

or

$$
\frac{1}{\pi} \int_{-1}^{1} \frac{\Psi_{2}(x, \eta)}{(\eta-y)} d \eta=f(x, y) \ln \frac{1-x}{1+x} \ln \frac{1-y}{1+y} .
$$

Solving the equation (13) in the class $h_{0}$, we obtain [9]

$$
\Psi_{1}(x, y)=-\frac{\ln \frac{1-y}{1+y}}{\sqrt{1-x^{2}}} \frac{1}{\pi} \int_{-1}^{1} \frac{\sqrt{1-\xi^{2}} \ln \frac{1-\xi}{1+\xi} f(\xi, y)}{(\xi-x)} d \xi+\frac{c_{1}(y)}{\sqrt{1-x^{2}}}
$$


where $c_{1}(y)$ is an arbitrary function from $h_{0}$.

Next, solving (11), we have

$$
\begin{aligned}
& \varphi(x, y) \\
& =\frac{R(f ; x, y)}{\sqrt{\left(1-x^{2}\right)\left(1-y^{2}\right)}}-\frac{1}{\sqrt{1-x^{2}}} \frac{1}{\pi} \int_{-1}^{1} \frac{\sqrt{1-\eta^{2}} c_{1}(\eta)}{\sqrt{1-y^{2}}(\eta-y)} d \eta+\frac{c_{2}(x)}{\sqrt{1-y^{2}}} \\
& =\frac{R(f ; x, y)}{\sqrt{\left(1-x^{2}\right)\left(1-y^{2}\right)}}+\frac{\gamma_{1}(x)}{\sqrt{1-y^{2}}}+\frac{\gamma_{2}(y)}{\sqrt{1-x^{2}}}
\end{aligned}
$$

where $R(f ; x, y)$ is given by the formula $(6)$, and $c_{2}(x), \gamma_{1}(x), \gamma_{2}(y)$ are arbitrary functions from $h_{0}$. We get the same result solving the equations (14) and, consequently, (12).

In order to determine the functions $\gamma_{1}(x), \gamma_{2}(y)$, we substitute the general solution given by (5) to the conditions (7)-(9). Then using the PoincaréBertrandt formula, we prove that the unique solution of the equation (1) in the class $h_{0} \times h_{0}$ is given by the formula (10).

3. Exact solution in the class $h(-1,1) \times h(-1,1)$.

Theorem 2. Let the function $f(x, y) \in h(-1,1) \times h(-1,1)$. Then the unique solution of the equation $(1)$ in the class $h(-1,1) \times h(-1,1)$ exists if and only if the following conditions:

$$
\begin{aligned}
& \frac{1}{\pi} \int_{-1}^{1} \frac{f(x, \eta)}{\sqrt{1-\eta^{2}}} \ln \frac{1-\eta}{1+\eta} d \eta=0, \\
& \frac{1}{\pi} \int_{-1}^{1} \frac{f(\xi, y)}{\sqrt{1-\xi^{2}}} \ln \frac{1-\xi}{1+\xi} d \xi=0,
\end{aligned}
$$

are fulfilled and it is given by the following formula:

$$
\begin{aligned}
& \varphi(x, y) \\
& =\sqrt{\left(1-x^{2}\right)\left(1-y^{2}\right)} \frac{1}{\pi^{2}} \iint_{D} \frac{f(\xi, \eta) \ln \frac{1-\xi}{1+\xi} \ln \frac{1-\eta}{1+\eta}}{\sqrt{\left(1-\xi^{2}\right)\left(1-\eta^{2}\right)}(\xi-x)(\eta-y)} d \xi d \eta .
\end{aligned}
$$

Proof. Similarly to the previous proof we introduce denotations (11), (12) and express the equation (1) in the form (13) or (14), respectively. Solving the equation (13) in the class of bounded functions, having the condition (18) fulfilled, we get [9]

$$
\Psi_{1}(x, y)=-\sqrt{1-x^{2}} \frac{1}{\pi} \int_{-1}^{1} \frac{f(\xi, y) \ln \frac{1-\xi}{1+\xi} \ln \frac{1-y}{1+y}}{\sqrt{1-\xi^{2}}(\xi-x)} d \xi .
$$


Next, from (14) and condition (17) we get

$$
\Psi_{2}(x, y)=-\sqrt{1-y^{2}} \frac{1}{\pi} \int_{-1}^{1} \frac{f(x, \eta) \ln \frac{1-x}{1+x} \ln \frac{1-\eta}{1+\eta}}{\sqrt{1-\eta^{2}}(\eta-y)} d \eta .
$$

It is easy to verify that the conditions (17), (18) are also sufficient for solvability of the equations (11) and (12), respectively in the class of bounded functions, and the solution of the equation (1) is given by the formula (19).

4. Approximate solution in the class $\boldsymbol{h}_{\mathbf{0}} \times \boldsymbol{h}_{\mathbf{0}}$. To find an approximate solution of the equation (1) in the class $h_{0} \times h_{0}$ we introduce a new unknown function $u(x, y)$ by the relation

$$
\begin{aligned}
& \frac{u(x, y)}{\sqrt{\left(1-x^{2}\right)\left(1-y^{2}\right)}} \\
& =\varphi(x, y)-\frac{g(x)}{\sqrt{1-y^{2}}}-\frac{h(y)}{\sqrt{1-x^{2}}}+\frac{A}{\sqrt{\left(1-x^{2}\right)\left(1-y^{2}\right)}} .
\end{aligned}
$$

Then it is easy to show that the problem (1), (7), (8), (9) takes the form

$$
\begin{gathered}
\frac{1}{\pi^{2}} \iint_{D} \frac{u(\xi, \eta)}{\sqrt{\left(1-\xi^{2}\right)\left(1-\eta^{2}\right)}} \frac{d \xi d \eta}{(\xi-x)(\eta-y)}=f(x, y) \ln \frac{1-x}{1+x} \ln \frac{1-y}{1+y} \\
\frac{1}{\pi} \int_{-1}^{1} \frac{u(x, \eta)}{\sqrt{1-\eta^{2}}} d \eta=0 \\
\frac{1}{\pi} \int_{-1}^{1} \frac{u(\xi, y)}{\sqrt{1-\xi^{2}}} d \xi=0 .
\end{gathered}
$$

Now we find an approximate solution of the problem (23), (24), (25). For this purpose we approximate the function $f(x, y)$ by the interpolating polynomial of the form

$$
f_{m n}(x, y)=\sum_{k=0}^{m} \sum_{j=0}^{n} f_{k j} x^{k} y^{j}
$$

and define the approximate solution $u_{m n}(x, y)$ as a solution of the following problem:

$$
\begin{aligned}
\frac{1}{\pi^{2}} \iint_{D} \frac{u_{m n}(\xi, \eta)}{\sqrt{\left(1-\xi^{2}\right)\left(1-\eta^{2}\right)}} \frac{d \xi d \eta}{(\xi-x)(\eta-y)} \\
=f_{m n}(x, y) \ln \frac{1-x}{1+x} \ln \frac{1-y}{1+y}
\end{aligned}
$$




$$
\begin{aligned}
& \frac{1}{\pi} \int_{-1}^{1} \frac{u_{m n}(x, \eta)}{\sqrt{1-\eta^{2}}} d \eta=0, \\
& \frac{1}{\pi} \int_{-1}^{1} \frac{u_{m n}(\xi, y)}{\sqrt{1-\xi^{2}}} d \xi=0 .
\end{aligned}
$$

To find the form of an approximate solution, we use the relation (22) and the exact solution in the class $h_{0} \times h_{0}$ given by (5) with the kernel (6). Next, substituting the function $f_{m, n}(x, y)$ defined in (26) in place of the given function $f(x, y)$ and using the formula

(30) $\frac{1}{\pi} \int_{-1}^{1} \sqrt{1-t^{2}} \ln \frac{1-t}{1+t} \frac{t^{k}}{t-x} d t=-\pi \sqrt{1-x^{2}} x^{k}-P_{k}(x),-1<x<1$,

we obtain

$$
\begin{aligned}
& u_{m n}(x, y) \\
& =\pi^{2} \sqrt{\left(1-x^{2}\right)\left(1-y^{2}\right)} f_{m n}(x, y)+\pi \sqrt{1-x^{2}} \sum_{k=0}^{m} \sum_{j=0}^{n} f_{k j} x^{k} Q_{j}(y) \\
& +\pi \sqrt{1-y^{2}} \sum_{k=0}^{m} \sum_{j=0}^{n} f_{k j} y^{j} P_{k}(x)+\sum_{k=0}^{m} \sum_{j=0}^{n} c_{k j} x^{k} y^{j}
\end{aligned}
$$

where $P_{k}(x), Q_{j}(y)$ are the polynomials of degree $k$ and $j$, respectively, defined as the principal part of the Laurent expansion of the following functions:

$$
\begin{aligned}
z^{k} \sqrt{z^{2}-1} \ln \frac{z-1}{z+1} & =P_{k}(z)+\frac{\widetilde{p}_{1}^{(k)}}{z}+\frac{\widetilde{p}_{2}^{(k)}}{z^{2}}+\ldots, \\
w^{j} \sqrt{w^{2}-1} \ln \frac{w-1}{w+1} & =Q_{j}(w)+\frac{\widetilde{q}_{1}^{(j)}}{w}+\frac{\widetilde{q}_{2}^{(j)}}{w^{2}}+\ldots,
\end{aligned}
$$

in a neighborhood of the infinity, $c_{k j}$ are the unknown coefficients.

To determine the coefficients $c_{k j}$ we substitute the right-hand side of the formula (31) to the equation (27), getting 


$$
\begin{aligned}
& \sum_{k=1}^{m} \sum_{j=1}^{n} c_{k j}\left(\frac{1}{\pi} \int_{-1}^{1} \frac{\xi^{k}}{\sqrt{1-\xi^{2}}} \frac{d \xi}{\xi-x}\right)\left(\frac{1}{\pi} \int_{-1}^{1} \frac{\eta^{j}}{\sqrt{1-\eta^{2}}} \frac{d \eta}{\eta-y}\right) \\
& =\sum_{k=0}^{m} \sum_{j=0}^{n} f_{k j} x^{k} y^{j} \ln \frac{1-x}{1+x} \ln \frac{1-y}{1+y} \\
& \quad-\sum_{k=0}^{m} \sum_{j=0}^{n} f_{k j}\left(\int_{-1}^{1} \frac{\xi^{k}}{\xi-x} d \xi\right)\left(\int_{-1}^{1} \frac{\eta^{j}}{\eta-y} d \eta\right) \\
& -\sum_{k=0}^{m} \sum_{j=0}^{n} f_{k j}\left(\int_{-1}^{1} \frac{\xi^{k}}{\xi-x} d \xi\right)\left(\frac{1}{\pi} \int_{-1}^{1} \frac{Q_{j}(\eta)}{\sqrt{1-\eta^{2}}} \frac{d \eta}{\eta-y}\right) \\
& -\sum_{k=0}^{m} \sum_{j=0}^{n} f_{k j}\left(\int_{-1}^{1} \frac{\eta^{j}}{\eta-y} d \eta\right)\left(\frac{1}{\pi} \int_{-1}^{1} \frac{P_{k}(\xi)}{\sqrt{1-\xi^{2}}} \frac{d \xi}{\xi-x}\right) .
\end{aligned}
$$

Denoting

(35) $P_{k}(z)=p_{0}^{(k)}+p_{1}^{(k)} z+\ldots+p_{k}^{(k)} z^{k}, \quad Q_{j}(z)=q_{0}^{(j)}+q_{1}^{(j)} z+\ldots+q_{j}^{(j)} z^{j}$, and taking into account the following formulae:

$$
\begin{aligned}
& \frac{1}{\pi} \int_{-1}^{1} \frac{t^{n}}{\sqrt{1-t^{2}}(t-x)} d t= \begin{cases}0, & n=0 \\
\sum_{k=1}^{\left[\frac{n+1}{2}\right]} \frac{2 k(2 k) !}{(2 k-1)(k !)^{2} 4^{k}} x^{n-2 k+1}, & n \geq 1\end{cases} \\
& \int_{-1}^{1} \frac{t^{n}}{t-x} d t= \begin{cases}\log \frac{1-x}{1+x}, & n=0, \\
\sum_{k=1}^{\left[\frac{n+1}{2}\right]} \frac{2}{2 k-1} x^{n-2 k+1}, & n \geq 1,\end{cases}
\end{aligned}
$$

we compare the corresponding coefficients getting formulae for coefficients $c_{k j}, k, j=1, \ldots, n$. Then substituting the right-hand side of the formula (31) to the conditions (28), (29), we get 


$$
\begin{aligned}
\sum_{k=0}^{m} \sum_{j=0}^{n} c_{k j}\left(\frac{1}{\pi} \int_{-1}^{1} \frac{\eta^{j}}{\sqrt{1-\eta^{2}}} d \eta\right) x^{k} \\
=-\pi \sqrt{1-x^{2}} \sum_{k=0}^{m} f_{k j}\left(\int_{-1}^{1} \eta^{j} d \eta\right) x^{k} \\
-\pi \sqrt{1-x^{2}} \sum_{k=0}^{m} \sum_{j=0}^{n} f_{k j}\left(\frac{1}{\pi} \int_{-1}^{1} \frac{Q_{j}(\eta)}{\sqrt{1-\eta^{2}}} d \eta\right) x^{k} \\
-\sum_{k=0}^{m} \sum_{j=0}^{n} f_{k j}\left(\int_{-1}^{1} \eta^{j} d \eta\right) P_{k}(x)
\end{aligned}
$$

and

$$
\begin{aligned}
\sum_{k=0}^{m} \sum_{j=0}^{n} c_{k j}\left(\frac{1}{\pi} \int_{-1}^{1} \frac{\xi^{k}}{\sqrt{1-\xi^{2}}} d \xi\right) y^{j} \\
=-\pi \sqrt{1-y^{2}} \sum_{k=0}^{m} \sum_{j=0}^{n} f_{k j}\left(\int_{-1}^{1} \xi^{k} d \xi\right) y^{j} \\
-\pi \sqrt{1-y^{2}} \sum_{k=0}^{m} \sum_{j=0}^{n} f_{k j}\left(\frac{1}{\pi} \int_{-1}^{1} \frac{P_{k}(\xi)}{\sqrt{1-\xi^{2}}} d \xi\right) y^{k} \\
\quad-\sum_{k=0}^{m} \sum_{j=0}^{n} f_{k j}\left(\int_{-1}^{1} \xi^{k} d \xi\right) Q_{j}(y),
\end{aligned}
$$

respectively. From the above equations, using formula

$$
\frac{1}{\pi} \int_{-1}^{1} \frac{t^{n}}{\sqrt{1-t^{2}}} d t= \begin{cases}0, & n=1,3,5, \ldots, \\ \frac{(n+2)(n+2) !}{(n+1)\left(\frac{n+2}{2} !\right)^{2} 2^{n+2}}, & n=0,2,4, \ldots,\end{cases}
$$

we get the coefficients $c_{00}, c_{0 j}, c_{k 0}, j, k=1, \ldots, n$.

Finally,

$$
c_{k j}=\sum_{r=k}^{m} \sum_{s=j}^{n} f_{r s} p_{k}^{(r)} q_{j}^{(s)}, \quad k=0,1, \ldots, m, j=0,1, \ldots, n,
$$

where $p_{k}^{(r)}, q_{j}^{(s)}$ are the coefficients of the polynomials $P_{r}(x), Q_{s}(y)$ given in (32) and (33). 
5. Approximate solution in the class $h(-1,1) \times h(-1,1)$. As previously, we introduce a new unknown function $u(x, y)$ defined by

$$
\varphi(x, y)=\sqrt{\left(1-x^{2}\right)\left(1-y^{2}\right)} u(x, y) .
$$

Then the equation (1) takes the form

(43) $\frac{1}{\pi^{2}} \iint_{D} \sqrt{\left(1-\xi^{2}\right)\left(1-\eta^{2}\right)} \frac{u(\xi, \eta) d \xi d \eta}{(\xi-x)(\eta-y)}=f(x, y) \ln \frac{1-x}{1+x} \ln \frac{1-y}{1+y}$,

where the given function $f(x, y)$ satisfies the conditions (17), (18). Next, we approximate the function $f(x, y)$ by the polynomial $(26)$ and define the approximate solution $u_{m n}(x, y)$ as a solution of the following equation:

$$
\begin{aligned}
& \frac{1}{\pi^{2}} \iint_{D} \sqrt{\left(1-\xi^{2}\right)\left(1-\eta^{2}\right)} \frac{u_{m n}(\xi, \eta) d \xi d \eta}{(\xi-x)(\eta-y)} \\
& \quad=f_{m n}(x, y) \ln \frac{1-x}{1+x} \ln \frac{1-y}{1+y}+Q_{1}^{*}(x)+Q_{2}^{*}(y) .
\end{aligned}
$$

Note that the function $f_{m n}(x, y)$ does not have to satisfy the conditions (17), (18). Therefore the sum $Q_{1}^{*}(x)+Q_{2}^{*}(y)$ is added. Substituting the right-hand side of the equation (44) to the conditions (17), (18), we get the relations

$$
\begin{aligned}
& \ln \frac{1-x}{1+x} \frac{1}{\pi} \int_{-1}^{1} \frac{f_{m n}(x, \eta) \ln \frac{1-\eta}{1+\eta}}{\sqrt{1-\eta^{2}}} d \eta+Q_{1}^{*}(x)+\frac{1}{\pi} \int_{-1}^{1} \frac{Q_{2}^{*}(\eta)}{\sqrt{1-\eta^{2}}} d \eta=0, \\
& \ln \frac{1-y}{1+y} \frac{1}{\pi} \int_{-1}^{1} \frac{f_{m n}(\xi, y) \ln \frac{1-\xi}{1+\xi}}{\sqrt{1-\xi^{2}}} d \xi+\frac{1}{\pi} \int_{-1}^{1} \frac{Q_{1}^{*}(\xi)}{\sqrt{1-\xi^{2}}} d \xi+Q_{2}^{*}(y)=0 .
\end{aligned}
$$

Dividing (45) by $\sqrt{1-x^{2}}$, integrating respect to $x$, and adding both sides of the equations (45) and (46), we get

$$
\begin{aligned}
Q_{1}^{*}(x)+Q_{2}^{*}(y)=\frac{1}{\pi^{2}} & \iint_{D} \frac{f_{m n}(\xi, \eta) \ln \frac{1-\xi}{1+\xi} \ln \frac{1-\eta}{1+\eta}}{\sqrt{\left(1-\xi^{2}\right)\left(1-\eta^{2}\right)}} d \xi d \eta \\
& -\ln \frac{1-x}{1+x} \frac{1}{\pi} \int_{-1}^{1} \frac{f_{m n}(x, \eta) \ln \frac{1-\eta}{1+\eta}}{\sqrt{1-\eta^{2}}} d \eta \\
& -\ln \frac{1-y}{1+y} \frac{1}{\pi} \int_{-1}^{1} \frac{f_{m n}(\xi, y) \ln \frac{1-\xi}{1+\xi}}{\sqrt{1-\xi^{2}}} d \xi
\end{aligned}
$$


Similarly to the class $h_{0} \times h_{0}$, it can be proved that the approximate solution $u_{m n}(x, y)$ has the form

$$
\begin{gathered}
u_{m n}(x, y)=\sum_{k=0}^{m-2} \sum_{j=0}^{n-2} c_{k j} x^{k} y^{j}+\frac{\pi^{2}}{\sqrt{\left(1-x^{2}\right)\left(1-y^{2}\right)}} f_{m n}(x, y) \\
-\frac{\pi}{\sqrt{1-x^{2}}} \sum_{k=0}^{m} \sum_{j=0}^{n-2} f_{k, j+2} x^{k} Q_{j}(y) \\
-\frac{\pi}{\sqrt{1-y^{2}}} \sum_{k=0}^{m-2} \sum_{j=0}^{n} f_{k+2, j} P_{k}(x) y^{j},
\end{gathered}
$$

where $P_{k-2}(x), Q_{j-2}(y)$ are polynomials of degree $k-2$ i $j-2$, respectively, defined as the leading part of the Laurent expansion of the following functions:

$$
\begin{aligned}
& \frac{z^{k}}{\sqrt{z^{2}-1}} \ln \frac{z-1}{z+1}=P_{k-2}(z)+\frac{\widetilde{p}_{1}^{(k)}}{z}+\frac{\widetilde{p}_{2}^{(k)}}{z^{2}}+\ldots, \\
& \frac{w^{j}}{\sqrt{w^{2}-1}} \ln \frac{w-1}{w+1}=Q_{j-2}(w)+\frac{\widetilde{q}_{1}^{(j)}}{w}+\frac{\widetilde{q}_{2}^{(j)}}{w^{2}}+\ldots
\end{aligned}
$$

in a neighborhood of the infinity. Here $c_{k j}$ are the unknown coefficients.

Substituting the right-hand side of the formula (48) to equation (44), we get

$$
\begin{aligned}
\sum_{k=0}^{m-2} \sum_{j=0}^{n-2} c_{k j}\left(\frac{1}{\pi} \int_{-1}^{1} \frac{\sqrt{1-\xi^{2}} \xi^{k}}{\xi-x} d \xi\right)\left(\frac{1}{\pi} \int_{-1}^{1} \frac{\sqrt{1-\eta^{2}} \eta^{j}}{\eta-y} d \eta\right) \\
+\sum_{k=0}^{m} \sum_{j=0}^{n} f_{k j}\left(\int_{-1}^{1} \frac{\xi^{k}}{\xi-x} d \xi\right)\left(\int_{-1}^{1} \frac{\eta^{j}}{\eta-y} d \eta\right) \\
-\sum_{k=0}^{m} \sum_{j=0}^{n-2} f_{k, j+2}\left(\int_{-1}^{1} \frac{\xi^{k}}{\xi-x} d \xi\right)\left(\frac{1}{\pi} \int_{-1}^{1} \frac{\sqrt{1-\eta^{2}} Q_{j}(\eta)}{\eta-y} d \eta\right) \\
-\sum_{k=0}^{m-2} \sum_{j=0}^{n} f_{k+2, j}\left(\int_{-1}^{1} \frac{\eta^{j}}{\eta-y} d \eta\right)\left(\frac{1}{\pi} \int_{-1}^{1} \frac{\sqrt{1-\xi^{2}} P_{k}(\xi)}{\xi-x} d \xi\right) \\
=f_{m n}(x, y) \ln \frac{1-x}{1+x} \ln \frac{1-y}{1+y}+Q_{1}^{*}(x)+Q_{2}^{*}(y),
\end{aligned}
$$


where $Q_{1}^{*}(x)+Q_{2}^{*}(y)$ is given by the formula (47). Using (37) and

$$
\begin{aligned}
& \frac{1}{\pi} \int_{-1}^{1} \frac{\sqrt{1-t^{2}} t^{k}}{t-x} d t=\sum_{k=0}^{\left[\frac{n+1}{2}\right]} \frac{(2 k) !}{(2 k-1)(k !)^{2} 4^{k}} x^{n-2 k+1}, \\
& \frac{1}{\pi} \int_{-1}^{1} \frac{t^{n} \log \frac{1-t}{1+t}}{\sqrt{1-t^{2}}} d t=-\operatorname{Res}_{z=\infty} \frac{z^{n} \ln \frac{z-1}{z+1}}{\sqrt{z^{2}-1}},
\end{aligned}
$$

we compare the coefficients and get

$$
c_{k j}=\sum_{r=k}^{m-2} \sum_{s=j}^{n-2} f_{r+2, s+2} p_{k}^{(r)} q_{j}^{(s)},
$$

$k=0,1, \ldots, m-2, j=0,1, \ldots, n-2$, where $p_{k}^{(r)}, q_{j}^{(s)}$ are the coefficients of the polynomials $P_{r}(x), Q_{s}(y)$ given in (49) and (50), respectively.

6. Example. We give the approximate solution of (1) in the class $h_{0} \times h_{0}$. Let

$$
f(x, y)=\frac{1}{\left(x^{2}-25\right)\left(y^{2}-16\right)}, g(x)=0, h(x)=0 .
$$

Then the exact solution $u(x, y)$ of the problem $(23)-(25)$ has the form

$$
u(x, y)=\frac{\left(\pi \sqrt{1-x^{2}}+2 \sqrt{6} \log \frac{2}{3}\right)\left(\pi \sqrt{1-y^{2}}+\sqrt{15} \log \frac{3}{5}\right)}{\left(x^{2}-25\right)\left(y^{2}-16\right)}
$$

and the problem $(1),(7),(8),(9)$ has the solution given by the function

$$
\varphi(x, y)=\frac{\left(\pi \sqrt{1-x^{2}}+2 \sqrt{6} \log \frac{2}{3}\right)\left(\pi \sqrt{1-y^{2}}+\sqrt{15} \log \frac{3}{5}\right)}{\sqrt{\left(1-x^{2}\right)\left(1-y^{2}\right)}\left(x^{2}-25\right)\left(y^{2}-16\right)} .
$$

The function $f(x, y)$ is approximated by the polynomial

$$
f(x, y) \approx f_{m n}(x, y)=\frac{1}{400} \sum_{p=1}^{m}\left(\frac{x}{5}\right)^{2 p} \sum_{q=1}^{n}\left(\frac{y}{4}\right)^{2 q} .
$$

Taking $m=n=10$, we compare values of the exact and approximate solutions for some points $(x, y) \in D$. The results are shown in Table 1 . 


\begin{tabular}{|c|c|c|}
\hline$x$ & $y$ & $u_{m n}(x, y)-u(x, y)$ \\
\hline 0.9999 & 0.9999 & $-2.8045206 \times 10^{-16}$ \\
\hline 0.8976 & 0.3504 & $2.8428276 \times 10^{-18}$ \\
\hline 0.4576 & 0.7234 & $-8.6580677 \times 10^{-18}$ \\
\hline 0.0026 & 0.0211 & $-4.9440984 \times 10^{-18}$ \\
\hline-0.0015 & 0.9986 & $1.32194482 \times 10^{-16}$ \\
\hline-0.5523 & 0.6686 & $-5.5595619 \times 10^{-18}$ \\
\hline-0.9853 & -0.0006 & $6.6776657 \times 10^{-18}$ \\
\hline-0.3247 & -0.8954 & $-3.0507894 \times 10^{-17}$ \\
\hline-0.0247 & -0.2354 & $-5.260122 \times 10^{-18}$ \\
\hline 0.4247 & -0.7554 & $-1.0698026 \times 10^{-17}$ \\
\hline 0.9487 & -0.1554 & $4.2669215 \times 10^{-18}$ \\
\hline
\end{tabular}

TABLE 1. Comparison of the exact and approximate solutions in the class $h_{0} \times h_{0}$.

The authors are now working on estimating errors of the approximate solutions. The results will be presented in the next papers.

\section{REFERENCES}

[1] Bisplinghoff, R. L., Ashley, H. and Halfman, R. L., Aeroelasticity, Dover Publications, Mineola, 1996.

[2] Gakhov, F. D., Boundary Value Problems, Nauka, Moscow, 1977.

[3] Karczmarek, P., Singular integral equation with a multiplicative Cauchy kernel in the half-plane, Opuscula Math. 28 (2008), 63-72.

[4] Karczmarek, P., Pylak, D., Wójcik, P., Singular integral equations with multiplicative Cauchy-type kernels, Fasc. Math. 50 (2013), in press.

[5] Lifanov, I. K., Singular Integral Equations and Discrete Vortices, VSP, Utrecht, 1996.

[6] Muskhelishvili, N. I., Singular Integral Equations. Boundary Problems of Function Theory and Their Application to Mathematical Physics, Dover Publications, Inc., Mineola, New York, 2008.

[7] Pylak, D., Approximate solutions of a singular integral equation with Cauchy kernel in the quarter plane, Opuscula Math. 28 (2008), 179-194.

[8] Pylak, D., Sheshko, M. A., Inversion of singular integrals with Cauchy kernels in the case of an infinite integration domain, Differ. Equ. 41 (2005), 1297-1310.

[9] Sheshko, M., Singular Integral Equations with Cauchy and Hilbert Kernels and Theirs Approximated Solutions, TN KUL, Lublin, 2003.

[10] Sheshko, M. A., Sheshko, S. M., Inversion of singular integrals with multiplicative Cauchy kernel and infinite integration domain, Differ. Equ. 47 (2011), 534-546. 
Paweł Wójcik

Institute of Mathematics and Computer Science

The John Paul II Catholic University of Lublin

Al. Racławickie 14

20-950 Lublin

Poland

e-mail: wojcikpa@kul.lublin.pl

Michail A. Sheshko

Institute of Mathematics and Computer Science

The John Paul II Catholic University of Lublin

Al. Racławickie 14

20-950 Lublin

Poland

e-mail: szeszko@kul.pl

Dorota Pylak

Institute of Mathematics and Computer Science The John Paul II Catholic University of Lublin

Al. Racławickie 14

20-950 Lublin

Poland

e-mail: bdorotab@kul.pl

Paweł Karczmarek

Institute of Mathematics and Computer Science

The John Paul II Catholic University of Lublin

Al. Racławickie 14

20-950 Lublin

Poland

e-mail: pawelk@kul.pl

Received September 1, 2011 